\title{
Polarization and SEDs from Microlensing of Circumstellar Envelopes
}

\section{Richard Ignace*}

Department of Physics, Astronomy, \& Geology, East Tennessee State University

E-mail: ignace@etsu.edu

\section{Jon E. Bjorkman}

Department of Physics and Astronomy, University of Toledo

E-mail: jon@physics.utoledo.edu

\section{Christina Bunker ${ }^{\dagger}$}

Department of Physics and Astronomy, SUNY Stony Brook

E-mail: christina.bunkeregmail.com

\begin{abstract}
Microlensing surveys have proven to be tremendously fruitful in providing valuable data products for many fields of astrophysics, from eclipse lightcurves for substellar candidates to limb darkening in stellar atmospheres. We report on a program of modeling observables from microlensing of circumstellar envelopes, particularly those of red giant stars that are the most likely to show finite source effects. We will summarize work for how polarization light curves can be used to infer envelope properties and will describe recent modeling of the time dependent spectral energy distributions (SEDs) for microlensing of dusty winds. One of the most exciting developments is the possibility of measuring variable polarization from microlensing in a suitable source using the RINGO polarimeter at La Palma. Also quite interesting is the possibility of probing a dusty wind using IRAC data for a suitable source in the event that Spitzer has a "warm" cycle.
\end{abstract}

The Manchester Microlensing Conference: The 12th International Conference and ANGLES Microlensing Workshop

January 21-25 2008

Manchester, $U K$

\footnotetext{
*Speaker.

${ }^{\dagger}$ Southeastern Association for Research in Astronomy (SARA) NSF-REU Summer Intern
} 


\section{Introduction}

Galactic microlensing has produced a number of results for stellar atmospheres as an important offshoot of the original intent to understand dark matter in the Galactic halo. Among these have been applications to stellar atmospheres (e.g., Gould 2001) such as limb darkening (e.g., Albrow et al. 1999, 2001; Fields et al. 2003; Abe et al. 2003; Cassan et al. 2006) and chromospheres in red giant stars (Afonso et al. 2001; Cassan et al. 2004; Thurl et al. 2006). The possibility for what amounts to mapping stellar atmospheres using microlensing arises when the lens and background source have a transit or near transit event. The more spectacular events, and consequently the ones most suitable for recovering the intensity profi le of the source atmosphere, occur for binary lenses when the source has a caustic crossing.

Researchers have been pushing the envelope of possibilities for studying stellar atmospheres through considerations of more complex effects that might be detectable. These include stellar rotation (Gould 1997), stellar spots (Heyrovsky \& Sasselov 2000; Han et al. 2000; Hendry et al. 2002), stellar pulsation (Bryce 2001), and differential stellar rotation (Hendry \& Ignace, in prep). In contrast, our group has focused largely on the prospects of constraining the structure of circumstellar envelopes through microlensing events.

There are several motivations for considering the influence of circumstellar emissions in the spectra and photometry from source transit events. First, the most likely targets to show fi nite source effects are red giant stars, and red giants have signifi cant winds. Their mass-loss rates $\dot{M}$ have values of around $10^{-10}$ to $10^{-8} M_{\odot} / \mathrm{yr}$, all the way to $10^{-5} M_{\odot} / \mathrm{yr}$ for rare asymptotic giant branch (AGB) stars (Dupree 1986, Willson 2000). Second, the opportunity to infer the intensity profi le of these sources through microlensing translates to mapping the wind flow structure. The red giants are interesting because the driving of their winds is still not well-understood. Certainly the AGB winds are dust-driven (Netzer \& Elitzur 1993). The lower $\dot{M}$ winds of red giants may be driven by some combination of molecular opacity and dust (Jorgensen \& Johnson 1992). Finally, an extended envelope simply means a larger source thus making a transit more likely, which may lead to additional constraints on the nature of the lens.

What kinds of questions about the atmospheres and winds of red giants could be addressed through microlensing? Although dust is known to play a role (even a dominant role in the case of AGBs) in the wind driving, dust condensation temperatures are typically below $2000 \mathrm{~K}$, whereas the red giant atmospheres are around $3000 \mathrm{~K}$ or more. This means dust formation occurs in the wind; however, the dust formation radius is not well-constrained observationally. Additionally, there is a well-known division in the Hertzsprung-Russell Diagram (HRD) known as the "LinskyHaisch dividing line" among giant stars between those that show X-ray emissions and those that do not (Linsky \& Haisch 1979); the cause for this division is still being considered (Suzuki 2007). Both the ionized chromospheres of red giants and the stellar winds with molecular or dust opacity offer scattering opacity sources that lead to polarization, hence our group has been exploring variable polarization signals that can arise from microlensing transit events. This contribution reviews the main results from the polarization calculations and prospects for detections and presents new results for the influence of microlensing on the spectral energy distributions (SEDs) of dusty wind sources. Our team has also studied emission line shape variations for lines that form in circumstellar envelopes (Ignace \& Hendry 1999; Bryce et al. 2003; Hendry et al. 2006). Such variations 


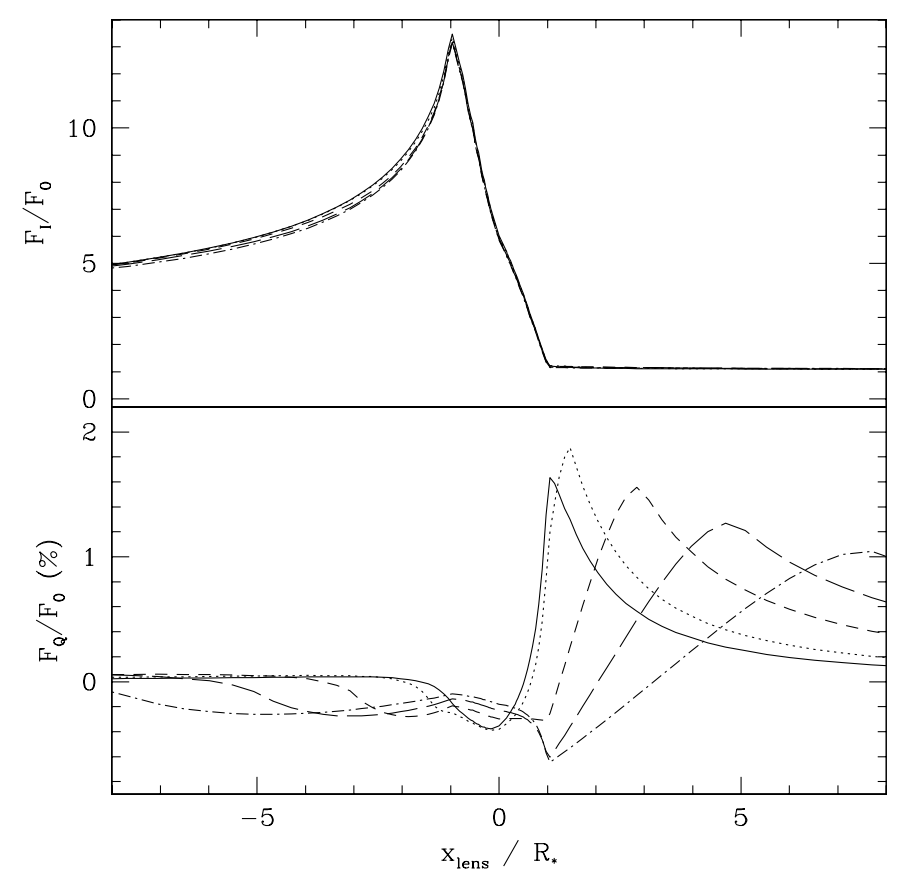

Figure 1: Stokes-I and Q fluxes normalized to the unlensed value $F_{0}$ and plotted for different positions relative to a straight line fold caustic (from Ignace et al. 2006). Negative values of $x_{\text {lens }}$ are interior to the caustic; positive are exterior. The Thomson scattering optical depth is $\tau_{\mathrm{sc}}=0.1$. Each curve is for a different hole radius $R_{\mathrm{h}}=1.0,1.5,3.0,5.0$, and $8.0 R_{*}$, with larger cavities giving variations spread over a larger $x_{\text {lens }}$ interval.

may be realized in appropriate lines for red giant winds or in supernovae; however, space does not permit a discussion of these models here.

\section{Variable Polarization from Microlensing}

For an unresolved but spherically symmetric source with scattering opacity, the total polarization will sum to zero. Although there is a non-zero polarized intensity along any given ray through the source medium, the polarizations from all the rays combine vectorially (e.g., using the Stokes notation $I, Q, U, V)$ such that the integration of contributions from all rays must sum to zero. A net polarization can only result from some asymmetry in the system, possibly a non-spherical envelope (Brown \& McLean 1977) or asymmetric illumination of scattering opacity (Al-Malki et al. 1999). Yet even in spherical symmetry, microlensing leads to a net polarization because it introduces an asymmetry into the system by virtue of selective magnifi cation.

Schneider \& Wagoner (1987) initiated ideas about variable polarization during microlensing events in their study of pseudo-photospheres of SNe. As galactic microlensing began to produce detections, and especially fi nite source events, Simmons et al. (1995ab) and Agol (1996) (and more recently Yoshida 2006) considered polarimetric signals from stellar atmospheres, while Simmons et al. (2002) and Ignace et al. (2006) have extended applications to circumstellar envelopes.

A key motivation for considering circumstellar envelopes is the possibility of determining the dust condensation radius. To explore such effects, Simmons et al. (2002) and Ignace et al. (2006) 
modeled the envelope as having an inner radius $\left(R_{\mathrm{h}}\right)$ for a "hole" of scattering opacity. Certainly gas exists between the photosphere at $R_{*}$ and $R_{\mathrm{h}}$, only that zone was assumed not to contribute to scattered light. Those models further assumed optically thin dipole scattering for simplicity. Example results for caustic crossing events are shown in Figure 11. The upper panel shows the normalized Stokes-I flux as the source moves from inside a straight line fold caustic to the outside. The lower panel shows the polarized emission. Note that the geometry arrangement ensures that Stokes- $U$ is zero. The change in sign of $Q$ indicates a position angle flip between parallel $(+)$ and perpendicular (-) orientations to the caustic line. Different curves are for different "hole radii" as labeled. It is clear that for binary lensing, quite large polarizations could result; however, this will depend on the envelope optical depth. In addition, the models in fi gure[1 ignore the potential for any polarization contribution from the photosphere or chromosphere, or molecular Rayleigh scattering that might exist in the "hole" region. All of these effects need to be explored more carefully using realistic stellar atmospheres.

It is worth noting that a general feature of polarization from microlensing is a measurement of the polarization position angle in the sky. This position angle provides information about the vector relative proper motion between the source and lens, information that in principle could prove useful in helping to constrain the lens location and its population.

It should also be pointed out that there is a claim for the detection of variable polarization from microlensing for a quasar (Chae et al. 2001). At the time of this writing, there has been no attempt to measure the effect in galactic microlensing; however, Iain Steele of the University of LiverpoolJohn Moores has agreed to provide discretionary time using the polarimeter RINGO (Steele et al. 2006) to measure broad band polarization in a suitable microlensing event.

\section{Variable SEDs from Dusty Envelopes}

Although dust may be present in the lower mass loss winds of typical red giants, it plays a dominant role in the wind driving for the high mass-loss rate AGB stars with $\dot{M} \approx 10^{-7} M_{\odot} / \mathrm{yr}$ or more. The presence of dust can alter or dominate the SEDs of red giants and AGB stars. A Monte Carlo radiative transfer algorithm has been used to model the SEDs of dusty winds during microlensing. The models assume spherical symmetry and a density profi le $\rho \propto r^{-2}$. Radiative equilibrium is enforced following Bjorkman \& Wood (2001). As a result, a self-consistent temperature profi le can be found for the dust. For the wind, the free parameters include the envelope optical depth $\tau_{V}$ (at $5500 \AA$ ) and the dust condensation radius $R_{\mathrm{h}}$. In principle, different grain species could also be considered; however, a standard interstellar dust opacity was adopted (Kim et al. 1994). The remaining free parameter is then the Einstein radius in the source plane $\hat{r}_{E}$ relative to $R_{\mathrm{h}}$.

Figure 1 illustrates the effect that dust can have on the stellar SED. There is no lensing in this example. The underlying stellar atmosphere is a Kurucz model at $T_{\text {eff }}=3500 \mathrm{~K}$ (1992) shown for the case of $\tau_{V}=0$. Also shown is a substantially dust-enshrouded star at $\tau_{V}=10$, displaying a radically different SED. The prominent IR bump near $10 \mu \mathrm{m}$ is dust silicate emission.

Key to understanding chromatic effects from microlensing transits is that the "effective" source size is a function of wavelength, $R_{\lambda}$. It is this effect that has allowed for the derivation of limb darkening from photometry and the probing of red giant chromospheres in $\mathrm{H} \alpha$. For a wind the density 


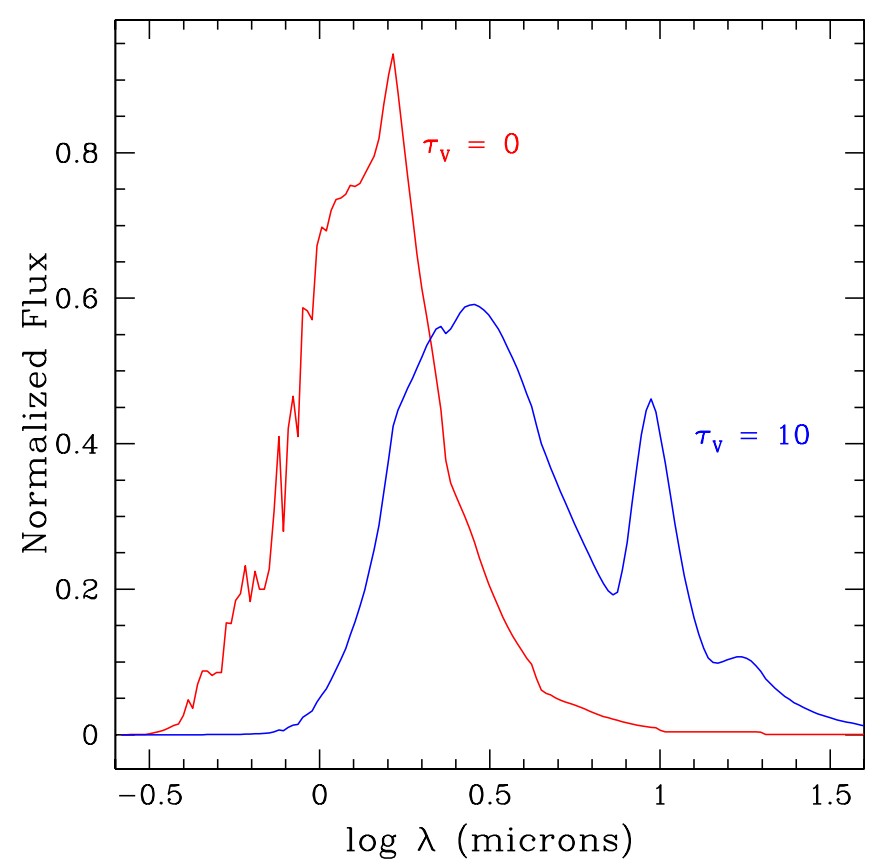

Figure 2: Left is the input Kurucz model spectrum appropriate for a red giant star; right shows the strongly redistributed emergent spectrum when this star has a thick dusty wind of $\tau_{V}=10$.

is generally power-law like, and the opacity is roughly power-law except in certain wavelength intervals. The combination can lead to a quite substantial range in $R_{\lambda}$ values as a function of wavelength.

Figure 3 displays one suite of model results for microlensing of dusty envelopes. This loglinear plot shows spectra in wavelength. The panels are for envelopes of different dust optical depths as labeled (again evaluated at $5500 \AA$ ). The unlensed spectrum is normalized to have unit area. Each curve is the lensed spectrum of the source at a different lens position (not labeled), from a distance of $30 R_{\mathrm{h}} / R_{*}$ down to $0.1 R_{\mathrm{h}} / R_{*}$, the latter corresponding to the mostly strongly magnifi ed spectrum in each panel. The lens position is not evenly spaced but staggered to approximate the rate of change in magnifi cation. Also note that the vertical scale is not the same for all three panels.

As expected, signifi cant dust content leads to a redistribution of the intrinsic stellar flux to the infrared (top panel). The density of spectral curves denotes the relative change in brightness as the event progresses. In other words one could take cuts at various wavelengths to obtain monochromatic lightcurves, and each one would tend to have a different maximum amplitude (relative to the unlensed case) and time width. For example in the top panel, the spectra shown for the IR "bump" near 10 microns are somewhat closely spaced. Here $R_{\lambda}$ is relatively large compared to $R_{*}$. A lightcurve at this wavelength will be broad in time and have a relatively depressed peak amplitude because $R_{\lambda} \gg \hat{r}_{E}$ (e.g., Agol 2003). In contrst, the spacing is more separated at NIR wavelengths. Here the source is more compact, and so variations between spectra vary more rapidly as the lens transits the source. In a light curve, the relative amplitude would be larger and the width more narrow in time. 


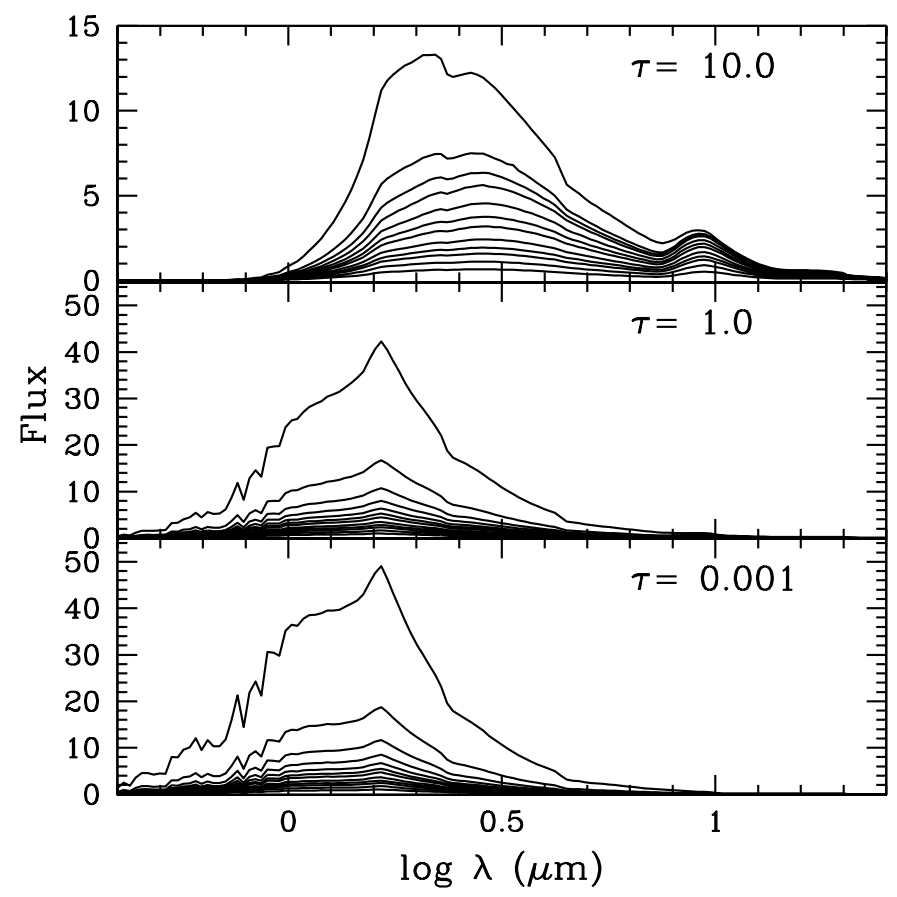

Figure 3: Simulated spectral variations for dusty envelopes with three different dust optical depths as indicated. Each has $R_{\mathrm{h}}=8 R_{*}$ and $\hat{E}=10 R_{\mathrm{h}}$. The unlensed spectrum is normalized to have unit wavelength integrated flux. Different curves are for different lens positions, with the most magnified cases corresponding to $x_{\text {lens }}=0.1 R_{\mathrm{h}}$.

Another way of displaying the results, and one more convenient for observers, is a color excess lightcurve. An example is displayed in Figure 4 Here model spectra were integrated for an R-band fi lter and for the Spitzer/IRAC fi lters at different lens positions *ns $/ R_{\mathrm{h}}$. The excess $E(R-I R A C n)$, for $n=1-4$, is relative to the unlensed value. The R-band samples the lightcurve for a relatively compact source at around 0.6 microns; the IRAC $n=1$ to 4 bandpasses sample different source sizes at 3.6, 4.5, 5.8, and $8.0 \mu \mathrm{m}$, respectively. As a result, the colors vary with differing amplitudes and widths as a function of lens location relative to the star (which of course can always be transformed to a lightcurve in time). $E(R-I R A C n)$ becomes positive ("redder") at early times because the source is relatively larger at longer wavelengths; then peaking occurs in the R-band as the lens nears the star because the source at that wavelength is spatially more compact. This information can be used to reconstruct the extent of the source size as a function of wavelength to test models of the density with radius in dusty winds. These effects will be stronger for winds of larger $\tau_{V}$ and should be measurable with Spitzer if there is a "warm" cycle.

The question arises, what is a suitable source? The result shown in Figure $\emptyset$ is for the case $\tau_{V}=1$, a substantial dust optical depth at an optical wavelength. One can relate a model optical depth to a stellar parameters. The optical depth is an integration through the wind along a radius. For $v_{\infty}$ the wind terminal speed of the wind, $\sigma=\rho_{\text {dust }} / \rho_{\text {wind }}$ the dust to gas ratio by mass, and $\kappa_{V}$ the dust opacity at $5500 \AA$, the optical depth is 


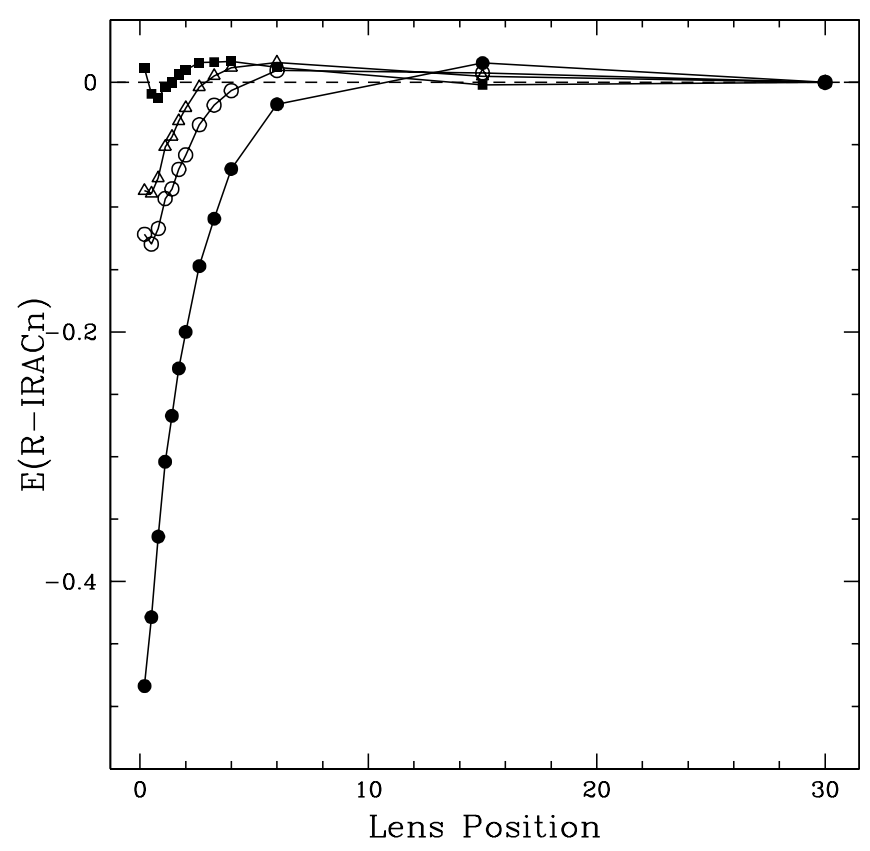

Figure 4: Simulated color excesses with lens position for ground-based R-band filter and Spitzer IRAC filters (squares for $3.6 \mu \mathrm{m}$, triangles for $4.5 \mu \mathrm{m}$, open circles for $5.8 \mu \mathrm{m}$, and filled circles for $8.0 \mu \mathrm{m}$ ). The envelope optical depth is $\tau_{V}=1$; the condensation radius is $R_{\mathrm{h}}=3 R_{*}$; and the Einstein radius in the source plane is $\hat{E}=10 R_{\mathrm{h}}$.

$$
\tau_{V}=0.001 \sigma \kappa_{V}\left(\frac{\dot{M}}{10^{-9} M_{\odot} / \mathrm{yr}}\right)\left(\frac{v_{\infty}}{30 \mathrm{~km} / \mathrm{s}}\right)^{-1}\left(\frac{R_{\mathrm{h}}}{24 R_{*}}\right)^{-1} g(w),
$$

where $g(w)$ allows for a wind velocity law (e.g., if $v / v_{\infty}=\sqrt{1-R_{\mathrm{h}} / r}$, then $g=2$ ).

For the ISM, $\sigma \approx 1 \%$ and $\kappa_{V} \approx 200 \mathrm{~cm}^{2} / \mathrm{g}$. All other factors being nominal and of order a few, this means that the wind mass-loss rate must be around $10^{-7} M_{\odot} / \mathrm{yr}$ for $\tau_{V}$ to much exceed unity. That level of mass loss is not achieved in red giants but only in AGB stars. Although rare, a couple of AGB stars have been observed as sources in microlensing events (Gaudi, priv. comm.); consequently, there is the possibility that chromatic effects arising from dust opacity in evolved cool star winds could be observable. Our early results only considered the point lens case. We can expect even more signifi cant chromatic variations from caustic crossing events with binary lenses.

\section{Acknowledgements}

This project was partially funded by a partnership between the National Science Foundation (NSF AST-0552798), Research Experiences for Undergraduates (REU), and the Department of Defense (DoD) ASSURE (Awards to Stimulate and Support Undergraduate Research Experiences).

\section{References}

[1] Abe F., et al., 2003, A\&A, 411, L493 
[2] Afonso, C., et al., 2001, A\&A, 378, 1014

[3] Albrow M.D., et al., 1999, ApJ, 522, 1011

[4] Agol E., 1996, MNRAS, 279, 571

[5] Agol E., 2003, ApJ, 594, 449

[6] Bjorkman, J., Wood, K., 2001, ApJ, 554, 615

[7] Brown J.C., McLean I.S., 1977, A\&A, 57, 141

[8] Bryce H.M., 2001, PhD Thesis, University of Glasgow

[9] Bryce H.M., Ignace R., Hendry M.A., 2003, A\&A, 401, 339

[10] Cassan, A., et al., 2004, A\&A, 419, L1

[11] Cassan, A., et al., 2006, A\&A, 460, 277

[12] Chae K.-H., et al., 2001, ApJ, 561, 653

[13] Dupree, A.K., 1986, ARAA, 24, 377

[14] Fields D.L., 2003, ApJ, 596, 1305

[15] Gould A., 1997, ApJ, 483, 98

[16] Gould A., 2001, PASP, 113, 903

[17] Han C., et al., 2000, MNRAS, 316, 665

[18] Hendry M.A., Bryce H.M., Valls-Gabaud D., 2002, MNRAS, 335, 539

[19] Hendry M.A., Ignace R., Bryce H.M., 2006, A\&A, 450, 1023

[20] Heyrovsky D., Sasselov D., 2000, Apj, 529, 69

[21] Ignace R., Hendry M.A., 1999, A\&A,341, 201

[22] Ignace R., Bjorkman J.E., Bryce H.M., 2006, MNRAS, 366, 921

[23] Jorgensen U.G., Johnson H.R., 1992, A\&A, 265, 168

[24] Kim S.-H., Martin P.G., Hendry P.D., 1994, ApJ, 422, 164

[25] Kurucz, R.L., 1992, in IAU Symposium 149, The Stellar Populations of Galaxies, ed. B. Barbuy \& A. Renzini (Dordrecht: Kluwer), 225

[26] Linsky J.L., Haisch, B.M., 1979, ApJ, 229, L27

[27] Netzer N., Elitzur M., 1993, ApJ, 410, 701

[28] Schneider P., Wagoner R.B., 1987, ApJ, 314, 154

[29] Simmons J., Willis J.P., Newsam A.M., 1995a, A\&A, 293, L46

[30] Simmons J., Newsam A.M., Willis J.P., 1995b, MNRAS, 276, 182

[31] Simmons J., et al., 2002, MNRAS, 336, 501

[32] Steele I.A., et al., 2006, SPIE, 6269, 62695

[33] Suzuki T., 2007, ApJ, 659, 1592

[34] Thurl, C., Sackett, P.D., Hauschildt, P.H., 2006, A\&A, 455, 315

[35] Willson, L.A., 2000, ARAA, 38, 573

[36] Yoshida H., 2006, MNRAS, 369, 997 